\title{
High-valence metals improve OER performance by modulating 3d metal oxidation cycle energetics
}

Bo Zhang ${ }^{1 \uparrow^{* *}}$, Lie Wang ${ }^{1 \dagger}$, Zhen $\mathrm{Cao}^{3 \dagger}$, Sergey M. Kozlov ${ }^{3}$, F. Pelayo García de Arquer ${ }^{2}$, Cao Thang Dinh ${ }^{2}$, Jun $\mathrm{Li}^{4}$, Ziyun Wang ${ }^{2}$, Xueli Zheng ${ }^{2}$, Longsheng Zhang1, Yunzhou Wen ${ }^{1}$, Oleksandr Voznyy ${ }^{2}$, Riccardo Comin ${ }^{2}$, Phil De Luna ${ }^{2}$, Tom Regier ${ }^{5}$, Wenli Bi ${ }^{6}$, E. Ercan Alp ${ }^{7}$, Chih-Wen $\mathrm{Pao}^{8}$, Lirong Zheng ${ }^{9}$, Yongfeng $\mathrm{Hu}^{5}$, Yujin $\mathrm{Ji}^{10}$, Youyong $\mathrm{Li}^{10}$, Ye Zhang ${ }^{11}$, Luigi Cavallo $^{3 *}$, Huisheng Peng ${ }^{1 *}$ and Edward H. Sargent ${ }^{2 *}$

${ }^{1}$ State Key Laboratory of Molecular Engineering of Polymers, Department of Macromolecular Science and Laboratory of Advanced Materials, Fudan University, Shanghai 200438, China

${ }^{2}$ Department of Electrical and Computer Engineering, University of Toronto, 35 St George Street, Toronto, Ontario M5S 1A4, Canada

${ }^{3}$ KAUST Catalysis Center, King Abdullah University of Science and Technology (KAUST), Thuwal 23955-6900, Saudi Arabia

${ }^{4}$ Institute of Chemical Sciences and Engineering, École polytechnique fédérale de Lausanne, Lausanne 1015, Switzerland

${ }^{5}$ Canadian Light Source, Inc.(CLSI), 44 Innovation Boulevard, Saskatoon, SK, S7N 2V3, Canada

${ }^{6}$ Department of Physics, University of Alabama at Birmingham, Birmingham, AL 35294, United States

${ }^{7}$ Advanced Photon Source, Argonne National Laboratory, 9700 S. Cass Avenue Argonne, Illinois, 60439, United States

${ }^{8}$ National Synchrotron Radiation Research Center, 101 Hsin Ann Road, Science-Based Industrial Park, Hsinchu, 30076, Taiwan

${ }^{9}$ Beijing Synchrotron Radiation Facility, Institute of High Energy Physics, Chinese Academy of Sciences, Beijing, 100049, China

${ }^{10}$ Institute of Functional Nano \& Soft Materials (FUNSOM) and Jiangsu Key Laboratory for Carbon-Based Functional Materials \& Devices, Soochow University, Suzhou, 215123, China

${ }^{11}$ College of Engineering and Applied Sciences, Nanjing University, Nanjing, 210023, China

†These authors contributed equally to this work

*Correspondence and requests for materials should be addressed to Edward H. Sargent (ted.sargent@utoronto.ca) (E.H.S), Huisheng Peng (penghs@fudan.edu.cn) (H.S.P), Bo Zhang (bozhang@fudan.edu.cn) (B.Z) and Luigi Cavallo (luigi.cavallo@kaust.edu.sa) (L.C). 
Multimetal oxyhydroxides have recently been reported that outperform noble metal catalysts for oxygen evolution reaction (OER). In such 3d-metal-based catalysts, the oxidation cycle of $3 \mathrm{~d}$ metals has been posited to act as the OER thermodynamic-limiting process; however, further tuning its energetics is challenging due to similarities among the electronic structures of neighboring $3 \mathrm{~d}$ metal modulators. Here we report a strategy to reprogram the $\mathrm{Fe}$, $\mathrm{Co}$, and $\mathrm{Ni}$ oxidation cycles by incorporating high-valence transitionmetal modulators $X(X=W, M o, N b, T a, R e$, and MoW). We use in-situlex-situ soft and hard $\mathrm{X}$-ray absorption spectroscopies to characterize the oxidation transition in modulated NiFeX and FeCoX oxyhydroxide catalysts, and conclude that the lower OER overpotential is facilitated by the readier oxidation transition of $3 d$ metals enabled by high-valence modulators. We report a $\sim 17$ fold mass activity enhancement compared with OER catalysts widely employed in industrial water-splitting electrolyzers. 
The oxygen evolution reaction (OER) - used at the anodic side in hydrogen evolution and carbon dioxide reduction systems - suffers from an excess voltage (overpotential) relative to that mandated by the thermodynamic value of the products ${ }^{1-9}$. Since the cost of renewable electricity will dominate the cost of renewable fuel production ${ }^{10}$, lowering the overpotentials contributes to lowering the cost of producing synthetic fuels ${ }^{11}$.

First-row (3d) transition-metal oxides are promising electrocatalysts for OER and are used in commercial electrolyzers ${ }^{6,9,12-15}$. The catalytic performance of multi-metal oxyhydroxides consisting of $3 \mathrm{~d}$ transition metals is improved by introducing additional elements that affect the electronic structure of the catalyst as a whole ${ }^{16-20}$, influencing the adsorption energies of intermediate species. This strategy was employed in ternary systems such as FeCoW metal oxyhydroxide to achieve catalysts that, with optimized $\mathrm{OH}^{*}, \mathrm{O}^{*}$, and $\mathrm{OOH}^{*}$ adsorption energies on the catalyst surface, exhibited OER performance superior to that of noble metal catalysts ${ }^{16}$.

It has been noted that in single-metal $\beta$-CoOOH frameworks, Co oxidation cycling through a $\mathrm{Co}^{3+} / \mathrm{Co}^{4+}$ transition is the thermodynamic limiting process (TLP), and the largest contributor to OER overpotential ${ }^{21}$. Modeling has predicted that facet manipulation in this framework can lead to a shift in cycling in $\mathrm{Co}^{3+} / \mathrm{Co}^{4+}$ toward lower valence of $\mathrm{Co}^{2+} / \mathrm{Co}^{3+} / \mathrm{Co}^{4+}$, in turn enabling lower overpotentials $^{21}$. This intriguing prediction has, however, not yet been proven in multinary $3 \mathrm{~d}$ metal oxyhydroxide systems. Previous work demonstrated the incorporation of high-valence $\mathrm{W}^{22}$, $\mathrm{Ta}^{22}$ and $\mathrm{Mo}^{23}$ to stabilize the otherwise-unstable low-charge $\mathrm{Fe}^{2+}$.

Here we posit a general doping strategy: the addition of metallic dopants with high-valence charges modulate $3 \mathrm{~d}$ metals ( $\mathrm{Fe} \mathrm{Co}$ and $\mathrm{Ni}$ ) toward lower energetics of valence charge transition, and hence better catalytic OER performance. We presented a physical model of OER in multinary metal oxyhydroxides, screened different dopants, and studied the modulation of $\mathrm{Fe} / \mathrm{Co} / \mathrm{Ni}$ valence 
states. We synthesized and characterized, using in-situ and ex-situ X-ray absorption spectroscopy (XAS), catalysts consisting of $\mathrm{NiFeX}$ and $\mathrm{FeCoX}$ (where $\mathrm{X}=\mathrm{W}, \mathrm{Mo}, \mathrm{Nb}, \mathrm{Ta}, \mathrm{Re}$, and MoW), and found good agreement with modeling results. As a result, we reported a 17 fold mass activity enhancement compared with the state-of-art catalysts in industrial hydrogen generation systems.

\section{Results}

\section{Density functional theory calculations}

We began by building a FeCo oxyhydroxide model based on an established computational framework $^{21}$ (Figure 1 and Supplementary Figure 1). The surface terminal groups were determined per the Pourbaix diagram (region (i) in Supplementary Figure 2), and the trend of electronic properties of the metal atoms within the $\mathrm{FeCoOOH}$ model was investigated via the Bader charge and magnetization (Figure 1a). Computational studies revealed that Co before OER exhibits $~ 3+$ charge, leading to a $3+/ 4+$ transition cycle during OER, corresponding to the TLP due to $\mathrm{OH}^{*}$ formation on the catalyst surface, which agreed well with literature ${ }^{21}$.

In computational studies, we doped high-valence metals into FeCo oxyhydroxides, forming FeCoX systems (X = Mo, W, Nb, Ta, and Re), and Co and Fe showed a lower charge state (see Figures 1a, Supplementary Figures 3 and 4, and Supplementary Table 1). The OER cycle of the FeCoX catalysts demonstrates a lower valence charge transition of Co active site (Figure 1c), in contrast with the conventional high valence charge transition cycle. FeCoX systems were found to enable the lowest overpotentials for OER in this system (Figure 1b and Supplementary Table 2). We ascribed this decrement in overpotential to the lower electron affinity of active sites in the FeCoX system compared to that of the FeCo control, which reduces the energy required to stimulate electron transfer. 
We extended the model to $\mathrm{NiFe}$ oxyhydroxide systems using the same metal dopants (Supplementary Figure 5), and the surface terminal groups were also determined per the Pourbaix diagram (region (i) in Supplementary Figure 6). We found that all studied NiFeX oxyhydroxides with higher valence charge dopants induced lower oxidation states of $\mathrm{Ni}$ and Fe than those in the pristine NiFe oxyhydroxide controls (Figure 1a, Supplementary Figures 7 and 8, and Supplementary Table 1), leading to a lower charge oxidation cycling of active sites during OER and therefore decreased overpotential (Figures $1 \mathrm{~b}$ and 1d and Supplementary Table 3).

Based on the fact that $\mathrm{X}=\mathrm{Mo}$ and $\mathrm{W}$ lead to the highest predicted activity, we further evaluated the overpotential of the quaternary metal oxyhydroxides, consisting of two best dopants together. It was found that the predicted activity can be further improved in FeCoX systems (FeCoMoW in Figure 1b).

\section{Materials synthesis and characterization}

We sought to investigate experimentally these concepts, preparing a suite of $\mathrm{NiFeX}$ and FeCoX oxyhydroxides and controls. We used a room-temperature sol-gel process ${ }^{16}$; after drying in vacuum, samples in the powder form were obtained (Supplementary Figure 9). High-resolution transmission electron microscopy (HRTEM), selected-area electron diffraction (SAED), energydispersive X-ray spectroscopy (EDS) mapping and X-ray diffraction (XRD) (Supplementary Figures 10-24) revealed an amorphous structure. TEM images (Supplementary Figures 10-23) and laser particle size analysis (Supplementary Figure 25) also show that these catalysts form agglomerated particles with sizes above $100 \mathrm{~nm}$. We further performed extended X-ray absorption fine structure (EXAFS) characterization to evaluate the distribution of different metals. Taking the FeCoMoW sample for example (Supplementary Figure 26), the Mo K-edge and W L3-edge EXAFS and fitting results indicate that peaks associated with $\mathrm{Mo}-\mathrm{O}-\mathrm{Co} / \mathrm{Fe}$ and $\mathrm{W}-\mathrm{O}-\mathrm{Co} / \mathrm{Fe}$ are 
presented. These suggest that Mo, W, and the $3 \mathrm{~d}$ metal in FeCoMoW sample were dispersed efficiently, which is in alignment with the structures we modeled, and is required to maximize the effect of modulation ${ }^{16}$.

To characterize the oxidation state of $3 \mathrm{~d}$ metal atoms in these materials before OER, we carried out ex-situ soft X-ray absorption spectroscopy (XAS) measurements monitoring 2p to $3 \mathrm{~d}$ transitions in 3d metals' L-edges in total electron yield (TEY) mode, which is surface-sensitive and valence-sensitive ${ }^{24,25}$. NiFe and FeCo samples (Figures $2 \mathrm{a}$ and $2 \mathrm{~b}$ ) yielded a significant amount of trivalent $\mathrm{Fe}^{3+}$. In contrast, NiFeMo, NiFeMoW, FeCoMo, and FeCoMoW exhibited a dominant amount of $\mathrm{Fe}^{2+}$ species with the main peak at $707.2 \mathrm{eV}$ and a shoulder peak at $718.7 \mathrm{eV}$ at $\mathrm{L}_{3}$ edge (Figures $2 \mathrm{a}$ and $2 \mathrm{~b}$ ). A series of time-dependent Fe L-edges XAS measurements were also performed to eliminate the possible beam damage from XAS to form the $\mathrm{Fe}^{2+}$ (Supplementary Note and Supplementary Figure 27). Thereafter, linear combination fitting of these spectra over two reference $\mathrm{Fe}$ oxides $\left(\mathrm{FeO}\right.$ for $\mathrm{Fe}^{2+}$ and $\mathrm{Fe}(\mathrm{OH})_{3}$ for $\mathrm{Fe}^{3+}$ ) allowed us to estimate the molar ratio of $\mathrm{Fe}^{2+}$ and $\mathrm{Fe}^{3+}$ (Supplementary Figures 28 and 29), with the results shown in Figure 2c and Supplementary Table 4. The high-valence metal modulated NiFe and FeCo samples exhibited higher $\mathrm{Fe}^{2+}$ percentage than those binary controls, in agreement with DFT predictions on the trend of oxidation states.

Furthermore, we measured the oxidation state of Fe using offline Mössbauer spectroscopy in transmission mode for FeCoMoW (Supplementary Figure 30) and hard XAS in Fe K-edge for NiFeMo (Supplementary Figure 31). Both Mössbauer spectra and K-edge X-ray absorption near edge structure (XANES) results showed significant $\mathrm{Fe}^{2+}$. After linear combination fitting with reference samples $\left(\mathrm{FeO}\right.$ for $\mathrm{Fe}^{2+}$ and $\mathrm{Fe}(\mathrm{OH})_{3}$ for $\mathrm{Fe}^{3+}$ ), the results show a decreased ratio of $\mathrm{Fe}^{2+}$ $(11 \%)$ in $\mathrm{FeCoMoW}$ and $\mathrm{Fe}^{2+}(20 \%)$ in NiFeMo compared to the Fe L-edge XAS results, a finding 
we ascribe to the fact that L-edge XAS is more surface-sensitive than that of K-edge XAS and Mössbauer spectroscopy ${ }^{25,26}$. This suggests that the high-valence-metal modulation on $3 \mathrm{~d}$ metals is more prominent on the catalyst surface, which can therefore tune the oxidation cycle energetics of $3 \mathrm{~d}$ metals and the adsorption energies of intermediate species during OER reaction ${ }^{16}$.

To assess the influence of high-valence metals on the oxidation transition of $3 \mathrm{~d}$ metals, we carried out in-situ Fe L-edge XAS under different applied potentials. First, we applied a bias of $+1.4 \mathrm{~V}$ at OER region to oxidize all $\mathrm{Fe}$ atoms to $\mathrm{Fe}^{3+}$ in both $\mathrm{FeCoMoW}$ and FeCo samples (Figure 2d). When the bias was changed to $+0.6 \mathrm{~V}(0.1 \mathrm{~V}$ lower than the thermodynamic equilibrium potential of $\left.\mathrm{Fe}^{3+} / \mathrm{Fe}^{2+}\right), \mathrm{Fe}^{3+}$ in $\mathrm{FeCoMoW}$ sample switched to a +2 oxidation state, while it did not change in FeCo sample. This suggests that the oxidation transition of Fe occurs easier in FeCoMoW than it does in the binary control, which is consistent with DFT calculations.

We further conducted in-situ Co K-edge (Figure 2e) and L-edge XAS (Supplementary Figure 32). At the initial state (before OER reaction) of all the studied FeCo based samples, Co is in a $\mathrm{Co}^{2+}$ configuration. At oxidizing bias during OER reaction, $\mathrm{Co}^{2+}$ species were fully oxidized to higher valence states in $\mathrm{FeCoMoW}$ sample, but they retained a $\mathrm{Co}^{2+}$ character in FeCo. Upon switching the applied potential from 1.5 to $1.2 \mathrm{~V}$ (Supplementary Figure 33), the oxidation state of Co changed to a low valence in FeCoMoW sample, which did not happen in FeCo sample. The ex-situ Co L-edge XAS spectra after OER reaction (Supplementary Figure 34) also showed consistent results. Furthermore, the oxidation transition of $\mathrm{Ni}$ in NiFe during OER reaction was also studied by in-situ Ni K-edge XAS (Figure 2f), and a similar conclusion was obtained.

The above XAS results indicate that the energy barriers of oxidation transition of $3 \mathrm{~d}$ metals are lower in high-valence-metal modulated systems than in binary controls. The previous studies have shown that $3 \mathrm{~d}$ metal oxides with a lower oxidation barrier exhibited increased OER 
activities $^{27-29}$. The present DFT studies also predicted that more facile oxidation transitions of $3 \mathrm{~d}$ metals lead to increased OER activity. We further characterized the oxidation of high-valence metal during OER: the results indicate that the oxidation state did not change (Supplementary Note and Supplementary Figure 35).

\section{Electrocatalytic performance}

We sought then to characterize the catalytic performance of the series of the $\mathrm{NiFeX}$ and FeCoX oxyhydroxides in the three-electrode configuration in $1 \mathrm{M} \mathrm{KOH}$ electrolytes at room temperature (see Methods). As shown in Figure 3, NiFeX and FeCoX exhibit lower overpotentials at $10 \mathrm{~mA} \cdot \mathrm{cm}^{2}$, an improvement of $37 \mathrm{mV}$ and $54 \mathrm{mV}$ compared with those of $\mathrm{NiFe}$ and FeCo catalysts, respectively (Supplementary Table 4). When comparing with prior best reports under the same testing conditions $\mathrm{s}^{30}$, the overpotential of NiFeMo is only $180 \mathrm{mV}$ at $10 \mathrm{~mA} \cdot \mathrm{cm}^{2}$ (Supplementary Figure 36).

To challenge further the posited link between $\mathrm{Fe}^{2+}$ and the catalytic performance for OER, we varied the composition and checked the relative concentrations of $\mathrm{Fe}^{2+}$ in each sample obtained using Fe L-edge XAS in TEY mode. For NiFe-based catalysts, the NiFeMo, NiFeW, NiFeNb, $\mathrm{NiFeTa}, \mathrm{NiFeRe}$, and $\mathrm{NiFeMoW}$ exhibited significantly higher $\mathrm{Fe}^{2+}$ ratio than that of the $\mathrm{NiFe}$ control, in agreement with simulation results (Figures 1 and 3e). For FeCo-based catalysts, a higher ratio of $\mathrm{Fe}^{2+}$ led to lower overpotentials (Figures 1 and 3f). Note that, although the catalysts with a higher $\mathrm{Fe}^{2+}$ content result in lower overpotentials, the correlation is not perfect and there are some exceptions. In addition, a possible correlation between dopants' covalent radii vs. performance was also investigated, and no clear correlation was found (Supplementary Figure37). 
To distinguish the effects of catalyst surface area vs. intrinsic performance, we investigated the turnover frequencies (TOFs). We used data obtained in $1 \mathrm{M} \mathrm{KOH}$ electrolyte at room temperature with $95 \%$ iR-correction at different potentials to calculate TOFs. NiFeMoW and FeCoMoW outperform control samples, exhibiting $\sim \times 17$ times and $\times 21$ times higher TOFs than binary $\mathrm{NiFe}$ and FeCo controls at $300 \mathrm{mV}$ overpotential, respectively (Figures $3 \mathrm{c}$ and $3 \mathrm{~d}$ ).

\section{Performance under industrial conditions}

We also carried out the same testing under industrial conditions $\left(30 \% \mathrm{KOH}\right.$ and at $\left.85^{\circ} \mathrm{C}\right)$, and observed trends that are consistent with those reported above (Supplementary Figure 38). The corresponding mass activities of the $\mathrm{NiFeX}$ and $\mathrm{FeCoX}$ based on the total loading mass of the ternary and quaternary oxides are enhanced compared with the NiFe and FeCo controls (Supplementary Figure 39). To characterize performance stability, we carried out water electrolysis by using NiFeMo catalysts and commercial Ru electrode as anode and cathode, respectively. The resultant electrolyser delivered $300 \mathrm{~mA} \cdot \mathrm{cm}^{-2}$ at $\sim 1.7 \mathrm{~V}$ consistently over $120 \mathrm{~h}$ (Supplementary Figure 40), indicating high stability of the catalysts. We also carried out water oxidation by cyclic voltammetry (CV) measurements at $50 \mathrm{mV} \cdot \mathrm{s}^{-1}$, and observed no appreciable decrease in the current densities during 2000 cycles (Supplementary Figure 41). To assess the performance of the catalysts under the industrial scale, we implemented these in industrial hydrogen generation systems (Figure 4a). The central part of the system is the electrolyzer (Figures $4 \mathrm{~b}$ and $4 \mathrm{c}$ ). Under various applied cell potentials, the current densities (normalized by loading mass) of the cell with the NiFeMo electrode are 17 times higher than the cell with a commercial Raney Ni electrode (Figure 4d). When compared at identical current density, NiFeMo also exhibits a lower cell voltage compared with Raney Ni catalysts. We observed no appreciable increase in the cell voltage during initial studies of 12-hour continuous operation (Figure 4e). 


\section{Conclusions}

In sum, this work describes how the incorporation of high-valence metal modulators promotes the catalytic activity of multinary $3 \mathrm{~d}$ metal compounds for OER. We offer a picture wherein the modulators lower the oxidation states of surface active sites (e.g. Fe, Co, and Ni) within their respective oxidation cycles. A synthetic route that seeks the homogeneous addition of such dopants, an approach based on a sol-gel process, enables charge redistribution across the catalyst. This allows the oxidation cycle of Fe, Co, and Ni sites to be optimized, under OER operation, toward a more energetically-favoured rate-limiting process. In-situ and ex-situ X-ray absorption spectroscopies suggest that $\mathrm{Fe}^{2+}$ facilitates the cycling of $\mathrm{Ni}$ and $\mathrm{Co}$ species between $2+$ and $3+$ oxidation states. The NiFeMo was used in the industrial electrolyzer system and exhibited $\sim 17$ times higher mass activity than commercial Raney Ni catalysts.

\section{Methods}

\section{Density functional simulations}

We used VASP to perform simulations with $\mathrm{rPBE}(\mathrm{GGA}=\mathrm{RP})$ exchange correlation functional augmented with Hubbard $U$ corrections for d-electrons taken from Materials project $(\mathrm{U}(\mathrm{Fe})=5.3 \mathrm{eV}, \mathrm{U}(\mathrm{Mo})=4.38 \mathrm{eV}, \mathrm{U}(\mathrm{W})=6.2 \mathrm{eV}, \mathrm{U}(\mathrm{Co})=3.32 \mathrm{eV}, \mathrm{U}(\mathrm{Ta})=2.3 \mathrm{eV}, \mathrm{U}(\mathrm{Re})=$ $2.7 \mathrm{eV}, \mathrm{U}(\mathrm{Nb})=1.3 \mathrm{eV})$. Valence electrons were described with a $400 \mathrm{eV}$ plane-wave basis set and $0.05 \mathrm{eV}$ Gaussian smearing of electronic density. Core electrons were described using the PAW method. We calculated 50 different spin states for each configuration and the discussion is based on the states that yield the lowest electronic energies. The employed slabs for FeCoX systems were constructed based on a 6-layer supercell of $\mathrm{CoOOH}$ with the lateral dimensions of $5.77 \times 9.31 \AA$. The reciprocal space was simulated using a $3 \times 3$ k-point mesh. The employed slabs for $\mathrm{NiFeX}$ systems were constructed based on a 6-layer supercell of $\mathrm{NiFeOOH}$ with a lateral 
dimension of $6.147 \times 12.459 \AA$. The reciprocal space was simulated using a $4 \times 2$ k-point mesh. The thermodynamic corrections to electronic energies were taken from literature ${ }^{16}$.

\section{Pourbaix diagram calculations}

Using the constructed model, we provide a systematic Pourbaix diagram calculation including over 3000 structural optimizations. We demonstrate surface coverage and the terminal groups for both FeCo and NiFe oxyhydroxides. For each type of oxyhydroxide, we counted the possible surface terminal groups, including the empty site, O-terminated, and $\mathrm{OH}$-terminated. For each configuration, we used ISPIN=2 in the VASP package to relax the magnetization. We then tested 50 different spin states near the relaxed magnetization using NUPDOWN in the VASP package and chose the configuration with the lowest energy. For instance, if the ISPIN $=2$ calculation gave magnetization $=70$, we re-made the optimization using all the 51 possibilities NUPDOWN $\in[45,95]$. In total, the Pourbaix diagram was obtained through 6630 calculations. The applied voltage corrections and $\mathrm{pH}$ corrections are performed using the following equation:

$$
\Delta G_{\text {corr }}=\Delta G-e U-0.059 p H
$$

where $\mathrm{U}$ is the applied voltage, and e is the electron transferred during the electrochemical reaction. The $\mathrm{pH}$ variation can be evaluated using the following chemical equation:

$$
\begin{aligned}
& *+\mathrm{H}_{2} \mathrm{O} \rightarrow \mathrm{O}^{*}+2 \mathrm{H}^{+}+2 e^{-} \\
& *+\mathrm{H}_{2} \mathrm{O}-\rightarrow \mathrm{OH}^{*}+\mathrm{H}^{+}+e^{-}
\end{aligned}
$$

The Pourbaix diagram calculation was made to obtain the surface coverage and the terminal groups for both FeCoX oxyhydroxides and NiFeX oxyhydroxides. The related results are provided as Supplementary Figures 2 and 6 for FeCo and NiFe systems, respectively.

\section{Chemicals}


Iron (III) chloride $\left(\mathrm{FeCl}_{3}\right)$, cobalt (II) chloride $\left(\mathrm{CoCl}_{2}\right)$, nickel chloride $\left(\mathrm{NiCl}_{2} \bullet 6 \mathrm{H}_{2} \mathrm{O}\right)$, tungsten (VI) chloride $\left(\mathrm{WCl}_{6}\right)$, molybdenum (V) chloride $\left(\mathrm{MoCl}_{5}\right)$, Niobium chloride $\left(\mathrm{NbCl}_{5}\right)$, Tantalum chloride $\left(\mathrm{TaCl}_{5}\right)$, Rhenium chloride $(\mathrm{ReCl} 5)$, ethanol $(\geq 99.5 \%)$, and propylene oxide ( $\geq 99 \%$ ) were purchased from Sigma-Aldrich. ${ }^{57} \mathrm{Fe}_{2} \mathrm{O}_{3}$ for preparing ${ }^{57} \mathrm{FeCl}_{3}$ was bought from Cyclotron Instruments. All the chemicals were used without further purification.

\section{Synthesis of multimetal oxyhydroxides}

All multimetal oxyhydroxides were synthesized using a modified aqueous sol-gel technique $^{16}$. For all the ternary mutltimetal oxyhydroxides, three types of metal chloride were used with identical mole. Taking NiFeMo for example, $\mathrm{NiCl}_{2} \cdot 6 \mathrm{H}_{2} \mathrm{O}(0.9 \mathrm{mmol})$, anhydrous $\mathrm{FeCl}_{3}(0.9$ $\mathrm{mmol})$ and $\mathrm{MoCl}_{5}(0.9 \mathrm{mmol})$ were first dissolved in ethanol $(4 \mathrm{~mL})$ in a vial. Then trace amount of deionized water $(0.21 \mathrm{ml})$ with ethanol $(2 \mathrm{ml})$ was prepared in another vial. After chilling, the solutions were mixed and propylene oxide $(1 \mathrm{ml})$ was slowly added to the mixed solution. Thereafter, a wet gel was formed. The wet-gel was aged for 24 hours and soaked in acetone for another 5 days. Then the wet gel was dried in a vacuum or with supercritical $\mathrm{CO}_{2}$ at room temperature. In terms of $\mathrm{FeCoMoW}$ or $\mathrm{NiFeMoW}$ sample, anhydrous $\mathrm{FeCl}_{3}(0.7 \mathrm{mmol}), \mathrm{CoCl}_{2}$ or $\mathrm{NiCl}_{2} \cdot 6 \mathrm{H}_{2} \mathrm{O}(0.7 \mathrm{mmol}), \mathrm{MoCl}_{5}(0.7 \mathrm{mmol})$ and $\mathrm{WCl}_{6}(0.7 \mathrm{mmol})$ were dissolved in ethanol (4 $\mathrm{mL}$ ) in a vial. The rest procedures are the same. $\mathrm{FeCo}, \mathrm{NiFe}$, and other ternary oxyhydroxides were synthesized following a process similar to that of NiFeMo oxyhydroxides.

\section{Synthesis of multimetal oxyhydroxides containing ${ }^{57} \mathrm{Fe}$}

${ }^{57} \mathrm{FeCl}_{3}$ was firstly prepared through dissolving ${ }^{57} \mathrm{Fe}_{2} \mathrm{O}_{3}$ in excess concentrated hydrochloric acid solution. Then the clear orange solution was evaporated via vacuum distillation until the slurry was formed, which was dissolved in ethanol. The rest steps are the same as above. 


\section{Characterization}

High-resolution transmission electron microscopy (HRTEM), selected area electron diffraction (SEAD), and energy-dispersive X-ray spectroscopy (EDS) were carried out using a Hitachi HF3300 at an accelerating voltage of $200 \mathrm{kV}$. The samples were prepared by dropping catalyst powder dispersed in ethanol onto carbon-coated copper TEM grids (Ted Pella, Redding, CA) using micropipettes and were dried under ambient conditions. The powder X-ray diffraction (XRD) patterns were measured on a Bruker D8 Advance spectrometer.

\section{XAS measurements}

Ex-situ sXAS measurements were performed at the Spherical Grating Monochromator (SGM) beamline of the Canadian Light Source, BL08U1-A beamlines at Shanghai Synchrotron Radiation Facility (SSRF) and 4B9B beamline of Beijing Synchrotron Radiation Facility (BSRF). All samples were scanned from 700 to $735 \mathrm{eV}$ in $0.1 \mathrm{eV}$ steps for the $\mathrm{Fe} \mathrm{L}_{2}, \mathrm{~L}_{3}$ and from 765 to $820 \mathrm{eV}$ in $0.1 \mathrm{eV}$ steps Co L2, L3 absorption edges. Surface sensitive absorption spectra were recorded using total electron yield (TEY).

In-situ sXAS measurements were performed at the SGM beamline of the Canadian Light Source. The window of the sample cells was mounted at an angle of roughly $45^{\circ}$ with respect to both the incident beam and the detectors. All measurements were made at room temperature in the fluorescence mode using Amptek silicon drift detectors (SDDs) with 1024 emission channels (energy resolution $\sim 120 \mathrm{eV}$ ). Four SDDs were employed simultaneously. For every edge, the scanning time was $30 \mathrm{~s}$ and repeated ten times, and the fluorescence of every edge was collected at the same absorption edge. The partial fluorescence yield (PFY) was extracted from all SDDs by the summation of the corresponding metal L emission lines. 
In-situ and ex-situ hard XAS measurements were performed at the $1 \mathrm{~W} 1 \mathrm{~B}$ beamline of the Beijing Synchrotron Radiation Facility (BSRF), BL14W1 beamline at the Shanghai Synchrotron Radiation Facility (SSRF), SuperXAS beamline at the Swiss Light Source and Taiwan Photon Source (TPS) 44A beamline. The In-situ experiment was conducted in a home-made triangular electrochemical cell, and working electrodes were prepared by loading catalyst samples on carbon paper. The spectra were obtained from 8.1 to $9.1 \mathrm{keV}$ for Ni K-edge XAS, 6.8 to $7.7 \mathrm{keV}$ for Fe K-edge XAS, 7.5 to $8.6 \mathrm{keV}$ for Co K-edge XAS, 19.8 to $20.8 \mathrm{keV}$ for Mo K-edge XAS, 10.0 to $11.0 \mathrm{keV}$ for $\mathrm{W} \mathrm{L}_{3}$-edge $\mathrm{XAS}$ and at $0.5 \mathrm{eV}$ steps at the near edge.

\section{Mössbauer spectroscopy measurements}

The Mössbauer experiments were done by off-line Mössbauer spectrometer in APS Mössbauer Laboratory (Advanced Photon Sources, Argonne National Laboratory) using ${ }^{57}$ Co source, not using synchrotron radiation (SR) source. The ${ }^{57} \mathrm{Co}$ source at the APS Mössbauer Laboratory was $10 \mathrm{mCi}$ and it was newly purchased. Data analysis was conducted using in-house software.

\section{Electrochemical measurements}

Electrochemical measurements were performed using a three-electrode configuration connected to Autolab PGSTAT302N and PGSTAT204N. The working electrode was carbon paper (TGP-H-060, TORAY) or Ni foam (thickness: $1.8 \mathrm{~mm}$ ). $\mathrm{Hg} / \mathrm{HgO}$ and platinum plates were used as reference and counter electrodes, respectively. To load the catalyst on working electrode, $10 \mathrm{mg}$ of catalyst was dispersed in $1 \mathrm{ml}$ of ethanol, followed by the addition of $80 \mu \mathrm{L}$ Nafion solution. The suspension was sonicated for $30 \mathrm{~min}$ to prepare a homogeneous ink. The catalytic electrode was prepared by spray coating the catalysts ink on carbon paper or Ni foam with a loading mass of about $1.67 \mathrm{mg} \cdot \mathrm{cm}^{-2}$. The area of the catalytic electrode was fixed to $0.5 \times 0.5 \mathrm{~cm}^{2}$ by coating 
with polyimide tape or water-resistant silicone glue for electrochemical testing. $\mathrm{CV}$ measurements at $50 \mathrm{mV} \cdot \mathrm{s}^{-1}$ were performed for 3 cycles before the record of linear scan voltammetry (LSV) at 5 $\mathrm{mV} \cdot \mathrm{s}^{-1}$ for each sample.

The performance tested under industrial condition (Supplementary Figures 26-29) were done on $\mathrm{Ni}$ foam at $30 \% \mathrm{KOH}$ solution with a temperature of $85 \pm 1{ }^{\circ} \mathrm{C}$. The industrial-scale measurements were performed using an electrolyzer at the Purification Equipment Research Institute of CSIC, China (http://www.peric718.com/Home/). A $10 * 10.5 \mathrm{~cm}^{2}$ NiFeMo electrode and commercial Ru electrode were used as an anode and cathode, respectively. Commercial Raney Ni electrode was used as a control.

We used electrochemical impedance spectroscopy (EIS) to determine uncompensated resistance $(R)$. The resistance values were 2.3 Ohms for NiFe, 2.4 Ohms for NiFeMo, 2.4 Ohms for NiFeW, 2.1 Ohms for NiFeTa, 2.6 Ohms for NiFeNb, 2.8 Ohms for NiFeRe, 2.4 Ohms for NiFeMoW, 2.5 Ohms for FeCo, 2.7 Ohms for FeCoW, 2.3 Ohms for FeCoMo, 2.2 Ohms for FeCoTa, 2.4 Ohms for FeCoNb, 2.6 Ohms for FeCoRe, and 2.1 Ohms for FeCoMoW on carbon paper electrode respectively. The resistance values were $0.37 \mathrm{Ohms}$ for $\mathrm{NiFe}, 0.38 \mathrm{Ohms}$ for NiFeMo, 0.37 Ohms for NiFeW, 0.35 Ohms for NiFeTa, 0.34 Ohms for NiFeNb, 0.36 Ohms for NiFeRe, 0.35 Ohms for NiFeMoW, 0.39 Ohms for FeCo, 0.37 Ohms for FeCoW, 0.36 Ohms for FeCoMo, 0.35 Ohms for FeCoTa, 0.36 Ohms for FeCoNb, 0.35 Ohms for FeCoRe, and 0.36 Ohms for FeCoMoW on Ni foam electrode respectively. At all potentials tested on carbon paper and $\mathrm{Ni}$ foam electrode, the potential was manually corrected using Ohm's law:

$$
E=E_{\text {applied }}-95 \% i R
$$

where Eapplied is applied potential, $i$ is the current measured, and compensating for $95 \%$ of the resistance $R$. 


\section{TOF calculations}

TOF is defined as the frequency of reaction on per active site, which is used to compare the intrinsic activity of different catalysts. For OER, TOF value is usually calculated by the equation:

$$
T O F=\frac{j \times A \times \eta}{4 \times F \times n}
$$

where $j$ is the current density after $95 \% i R$-compensation. $A$ is the geometric area of the electrode. $\eta$ is the Faradic efficiency and $F$ is Faraday's constant. $n$ is the molar number of active site. In our study, we assumed $\mathrm{Ni}$ and $\mathrm{Co}$ as active sites for $\mathrm{NiFeX}$ and FeCoX catalysts, and the number of $n$ were estimated via the total loading mass, according to equation:

$$
n=\frac{m \times N_{A}}{M w}
$$

where $m$ is the loading mass, $N_{A}$ is Avogadro's constant and $M w$ is the molecular weight of catalysts.

\section{Data availability}

The data that support the findings of this study are available in the Zenodo platform (DOI: 10.5281/zenodo.4008830) (ref. 31) ${ }^{31}$

\section{References}

1 Zhang, J., Zhao, Z., Xia, Z. \& Dai, L. A metal-free bifunctional electrocatalyst for oxygen reduction and oxygen evolution reactions. Nat. Nano. 10, 444-452 (2015).

$2 \mathrm{Ng}, \mathrm{J}$. W. D. et al. Gold-supported cerium-doped $\mathrm{NiO}_{\mathrm{x}}$ catalysts for water oxidation. Nat. Energy 1, 16053 (2016). 
3 Liang, Y. et al. $\mathrm{Co}_{3} \mathrm{O}_{4}$ nanocrystals on graphene as a synergistic catalyst for oxygen reduction reaction. Nat. Mater. 10, 780-786 (2011).

4 Seh, Z. W. et al. Combining theory and experiment in electrocatalysis: Insights into materials design. Science 355, eaad4998 (2017).

5 Seitz, L. C. et al. A highly active and stable $\mathrm{IrO}_{x} / \mathrm{SrIrO}_{3}$ catalyst for the oxygen evolution reaction. Science 353, 1011-1014 (2016).

6 Bergmann, A. et al. Unified structural motifs of the catalytically active state of Co(oxyhydr)oxides during the electrochemical oxygen evolution reaction. Nat. Catal. 1, 711-719 (2018).

7 Guan, J. et al. Water oxidation on a mononuclear manganese heterogeneous catalyst. Nat. Catal. 1, 870-877 (2018).

8 Martin-Sabi, M. et al. Redox tuning the Weakley-type polyoxometalate archetype for the oxygen evolution reaction. Nat. Catal. 1, 208-213 (2018).

9 Roy, C. et al. Impact of nanoparticle size and lattice oxygen on water oxidation on $\mathrm{NiFeO}_{x} \mathrm{H}_{\mathrm{y}}$. Nat. Catal. 1, 820-829 (2018).

10 Jouny, M., Luc, W. \& Jiao, F. High-rate electroreduction of carbon monoxide to multicarbon products. Nat. Catal. 1, 748-755 (2018).

11 Jouny, M., Luc, W. \& Jiao, F. General Techno-Economic Analysis of CO2 Electrolysis Systems. Ind. Eng. Chem. Res. 57, 2165-2177 (2018).

12 Galán-Mascarós, J. R. Water oxidation at electrodes modified with earth-abundant transition-metal catalysts. ChemElectroChem 2, 37-50 (2015).

13 Subbaraman, R. et al. Trends in activity for the water electrolyser reactions on 3d M(Ni,Co,Fe,Mn) hydr(oxy)oxide catalysts. Nat. Mater. 11, 550-557 (2012).

14 Roger, I., Shipman, M. A. \& Symes, M. D. Earth-abundant catalysts for electrochemical and photoelectrochemical water splitting. Nat. Rev. Chem. 1, 0003 (2017).

15 Fabbri, E. et al. Dynamic surface self-reconstruction is the key of highly active perovskite nano-electrocatalysts for water splitting. Nat. Mater. 16, 925-931 (2017).

16 Zhang, B. et al. Homogeneously dispersed multimetal oxygen-evolving catalysts. Science 352, 333-337 (2016). 
17 Rosalbino, F., Delsante, S., Borzone, G. \& Scavino, G. Electrocatalytic activity of crystalline $\mathrm{Ni}-\mathrm{Co}-\mathrm{M}(\mathrm{M}=\mathrm{Cr}, \mathrm{Mn}, \mathrm{Cu})$ alloys on the oxygen evolution reaction in an alkaline environment. Int. J. Hydrogen Energy 38, 10170-10177 (2013).

18 Chen, J. Y. C., Miller, J. T., Gerken, J. B. \& Stahl, S. S. Inverse spinel $\mathrm{NiFeAlO}_{4}$ as a highly active oxygen evolution electrocatalyst: promotion of activity by a redox-inert metal ion. Energy Environ. Sci. 7, 1382-1386 (2014).

19 Gerken, J. B., Shaner, S. E., Masse, R. C., Porubsky, N. J. \& Stahl, S. S. A survey of diverse earth abundant oxygen evolution electrocatalysts showing enhanced activity from Ni-Fe oxides containing a third metal. Energy Environ. Sci. 7, 2376-2382 (2014).

20 Friebel, D. et al. Identification of highly active $\mathrm{Fe}$ sites in (Ni,Fe)OOH for electrocatalytic water splitting. J. Am. Chem. Soc. 137, 1305-1313 (2015).

21 Bajdich, M., García-Mota, M., Vojvodic, A., Nørskov, J. K. \& Bell, A. T. Theoretical investigation of the activity of cobalt oxides for the electrochemical oxidation of water. $J$. Am. Chem. Soc. 135, 13521-13530 (2013).

22 Novák, M. et al. Primary oxide minerals in the system $\mathrm{WO}_{3}-\mathrm{Nb}_{2} \mathrm{O}_{5}-\mathrm{TiO}_{2}-\mathrm{Fe}_{2} \mathrm{O}_{3}-\mathrm{FeO}$ and their breakdown products from the pegmatite No. 3 at Dolní Bory-Hatě, Czech Republic. Eur. J. Mineral. 20, 487-499 (2008).

23 Kuepper, K. et al. Electronic and magnetic properties of highly ordered $\mathrm{Sr}_{2} \mathrm{FeMoO}_{6}$. Phys. Stat. Sol. (a) 201, 3252-3256 (2004).

24 Liu, X., Yang, W. \& Liu, Z. Recent progress on synchrotron-based in-situ soft X-ray spectroscopy for energy materials. Adv. Mater. 26, 7710-7729 (2014).

25 de Groot, F. M. F. et al. 1s2p resonant inelastic X-ray scattering of iron oxides. J. Phys. Chem. B 109, 20751-20762 (2005).

26 Mitsui, T. in Magmas Under Pressure (eds Yoshio Kono \& Chrystèle Sanloup) 179210 (Elsevier, 2018).

27 Zheng, X. et al. Theory-driven design of high-valence metal sites for water oxidation confirmed using in situ soft X-ray absorption. Nat. Chem. 10, 149-154 (2018).

28 Liu, P. F., Yang, S., Zheng, L. R., Zhang, B. \& Yang, H. G. Mo ${ }^{6+}$ activated multimetal oxygen-evolving catalysts. Chem. Sci. 8, 3484-3488 (2017). 
29 Liu, P. F., Yang, S., Zheng, L. R., Zhang, B. \& Yang, H. G. Electrochemical etching of $\alpha$-cobalt hydroxide for improvement of oxygen evolution reaction. J. Mater. Chem. A $\mathbf{4}$, 9578-9584 (2016).

30 Qiu, Z., Tai, C.-W., Niklasson, G. A. \& Edvinsson, T. Direct observation of active catalyst surface phases and the effect of dynamic self-optimization in NiFe-layered double hydroxides for alkaline water splitting. Energy Environ. Sci. 12, 572-581 (2019).

31 Zhang, B. et al. High-valence metals improve OER performance by modulating $3 \mathrm{~d}$ metal oxidation cycle energetics. Zenodo Digital Repository, 10.5281/zenodo.4008830 (2020).

\section{Acknowledgements}

This work was supported by MOST (2016YFA0203302), NSFC (21875042, 21634003, 51573027), STCSM (16JC1400702, 18QA1400800), SHMEC (2017-01-07-00-07-E00062) and Yanchang Petroleum Group. This work was also supported by The Program for Professor of Eastern Scholar at Shanghai Institutions of Higher Learning. This work was supported by the Ontario Research Fund-Research Excellence Program; NSERC; and the CIFAR Bio-Inspired Solar Energy program. This work has also benefited from SGM beamlines at Canadian Light Source; 1W1B and 4B9B beamlines at the Beijing Synchrotron Radiation Facility; BL14W1, BL08U1-A beamline at Shanghai Synchrotron Radiation Facility; and 44A beamline at Taiwan Photon Source (TPS). Mössbauer spectroscopy measurements were conducted at the Advanced Photon Source, a Department of Energy (DOE) Office of Science User Facility operated for the DOE Office of Science by Argonne National Laboratory under Contract DE-AC02-06CH11357. The authors acknowledge the Paul Scherrer Institut, Villigen, Switzerland, for provision of synchrotron radiation beamtime at beamline SuperXAS of the SLS and would like to thank Dr. Maarten Nachtegaal for assistance. The authors thank Max García-Melchor and Ying Zhang for the discussion of DFT calculations. The authors thank Jianghua Wu for the assistance of TEM 
measurements. The authors thank R. Wolowiec and D. Kopilovic for their assistance. For computer time, this research used the resources of the Supercomputing Laboratory at KAUST.

\section{Author contributions}

E.H.S., H.S.P., B.Z., and L.C. supervised the project. B.Z. designed the project. L.W. and B.Z. carried out the experiments. Z.C., S.M.K., and Z.W. carried out DFT simulations. L.W., X.L.Z., L.S.Z., Y.Z.W., C.W.P., L.R.Z. and J.L. carried out XAS measurements. T.R. assisted in-situ XAS experiments. L.W., F.P.G.A., R.C., J.L. performed the XAS results analysis. O.V., Z.Y.W., and P.D.L assisted DFT simulations. W.B. and E.E.A. carried out the Mössbauer spectroscopy experiment and data analysis. C.T.D. and Y.F.H. assisted the discussion of experiments. Y.J.J. and Y.Y.L. assisted the discussion of DFT simulations. Y.Z. assisted in TEM and XRD measurements. B.Z., L.W., Z.C., F.P.G.A., S.M.K., H.S.P., and E.H.S. wrote the manuscript. All authors discussed the results and assisted during manuscript preparation.

\section{Completing interests}

The authors declare no competing interests. 

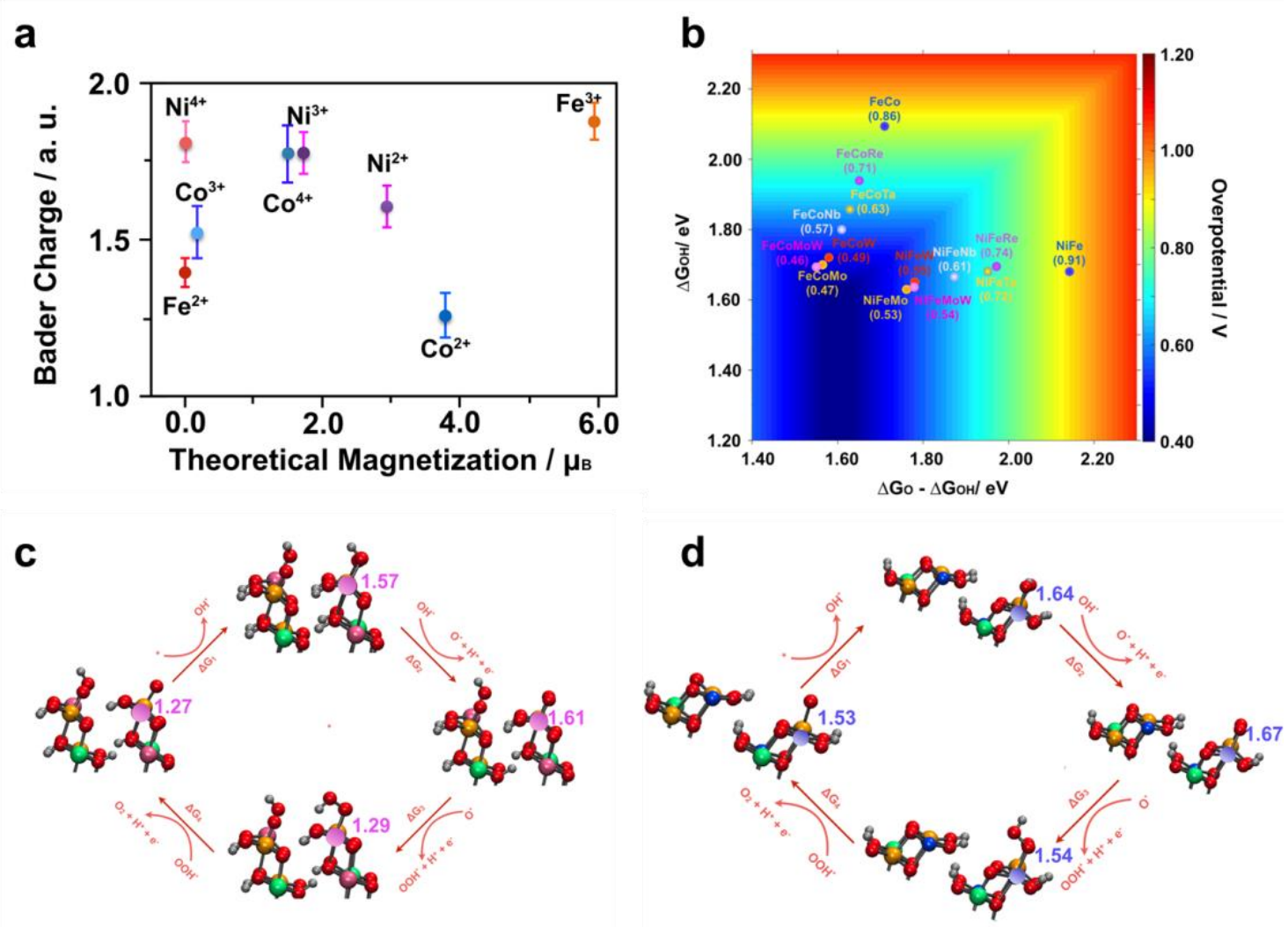

Figure 1. Density functional simulation findings. (a) Statistical analysis of the calculated Bader charge and magnetization obtained for $\mathrm{Fe}, \mathrm{Co}$, and $\mathrm{Ni}$ within the oxyhydroxide framework from 200 different configurations. The computed relative magnetizations for each component are consistent with the values predicted from crystal field theory for an octahedral environment. The error bar was calculated as the standard deviation: $s=\sqrt{\sum_{i=1}^{n}\left(x_{i}-\bar{x}\right)^{2} /(n-1)}$; (b) 2D map of the overpotentials generated by assuming $\Delta \mathrm{EoOH}=\mathrm{EoH}+3.2^{21}$, for different dopants in $\mathrm{FeCoX}$ and $\mathrm{NiFeX}$ catalysts: the overpotential can be reduced significantly with the high-valence charge metals; (c) and (d) demonstrate the OER cycles for the FeCoX and NiFeX, respectively, with the terminal group determined by the Pourbaix calculations (pink: Co; orange: $\mathrm{Fe}$; blue: Ni; green: high-valence metal; red: O; grey: H), and the Bader charge on the active metal site is listed. 

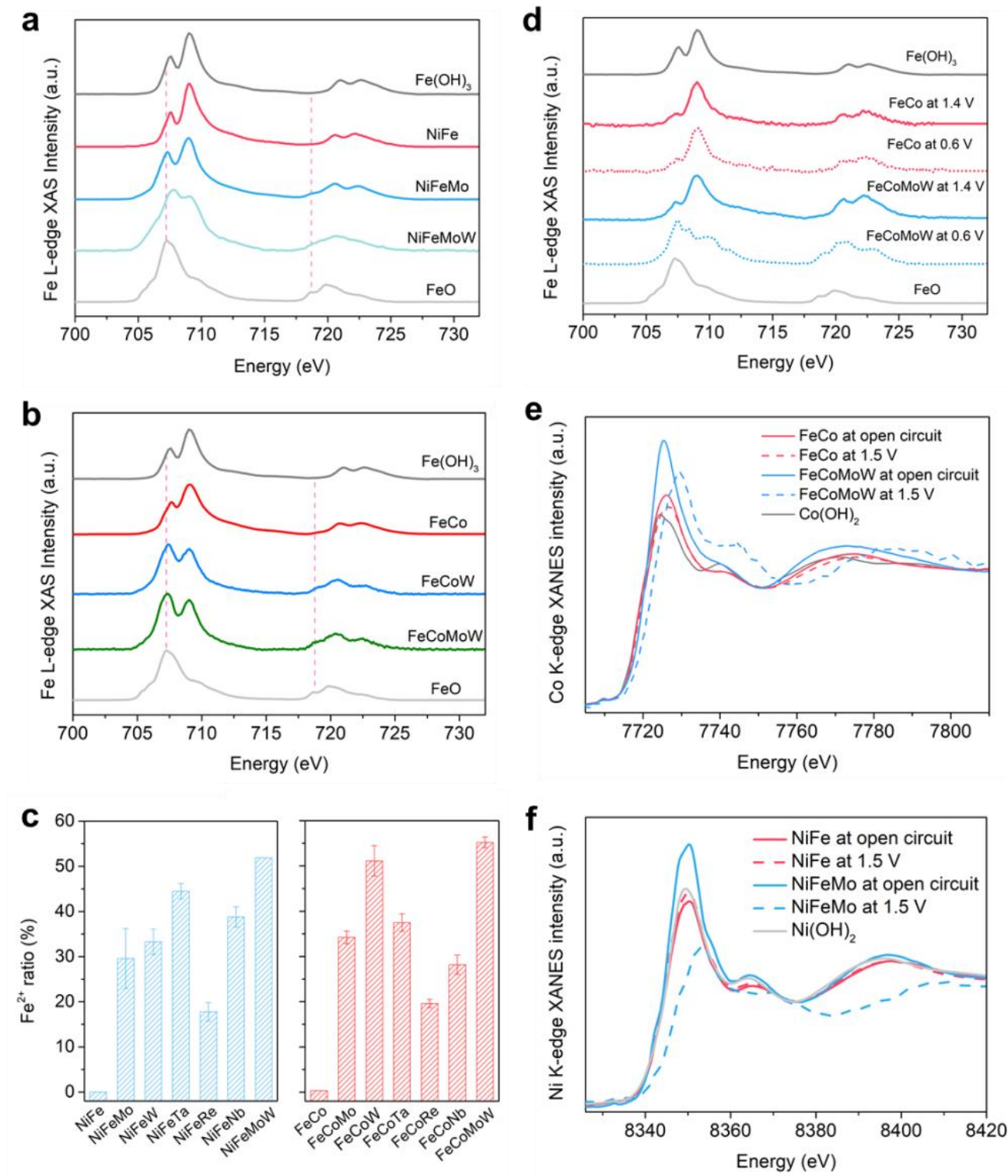

Figure 2. Oxidation state transition of $3 d$ metal in modulated catalysts. (a) TEY sXAS scans of NiFe, NiFeMo, and NiFeMoW at the Fe L-edge. (b) TEY sXAS scans of FeCo, FeCoW, and $\mathrm{FeCoMoW}$ at the Fe L-edge. (c) Concentration of $\mathrm{Fe}^{2+}$ species in all $\mathrm{NiFeX}$ and $\mathrm{FeCoX}$ samples. $\mathrm{Fe}^{2+}$ data is obtained by linear combination analysis of Fe L-edge TEY sXAS results. (d) The insitu Fe L-edge TEY sXAS spectra of FeCo and FeCoMoW. (e) The in-situ Co K-edge XANES spectra of FeCo and FeCoMoW. (f) In--situ Ni K-edge XANES spectra of NiFe and NiFeMo. 

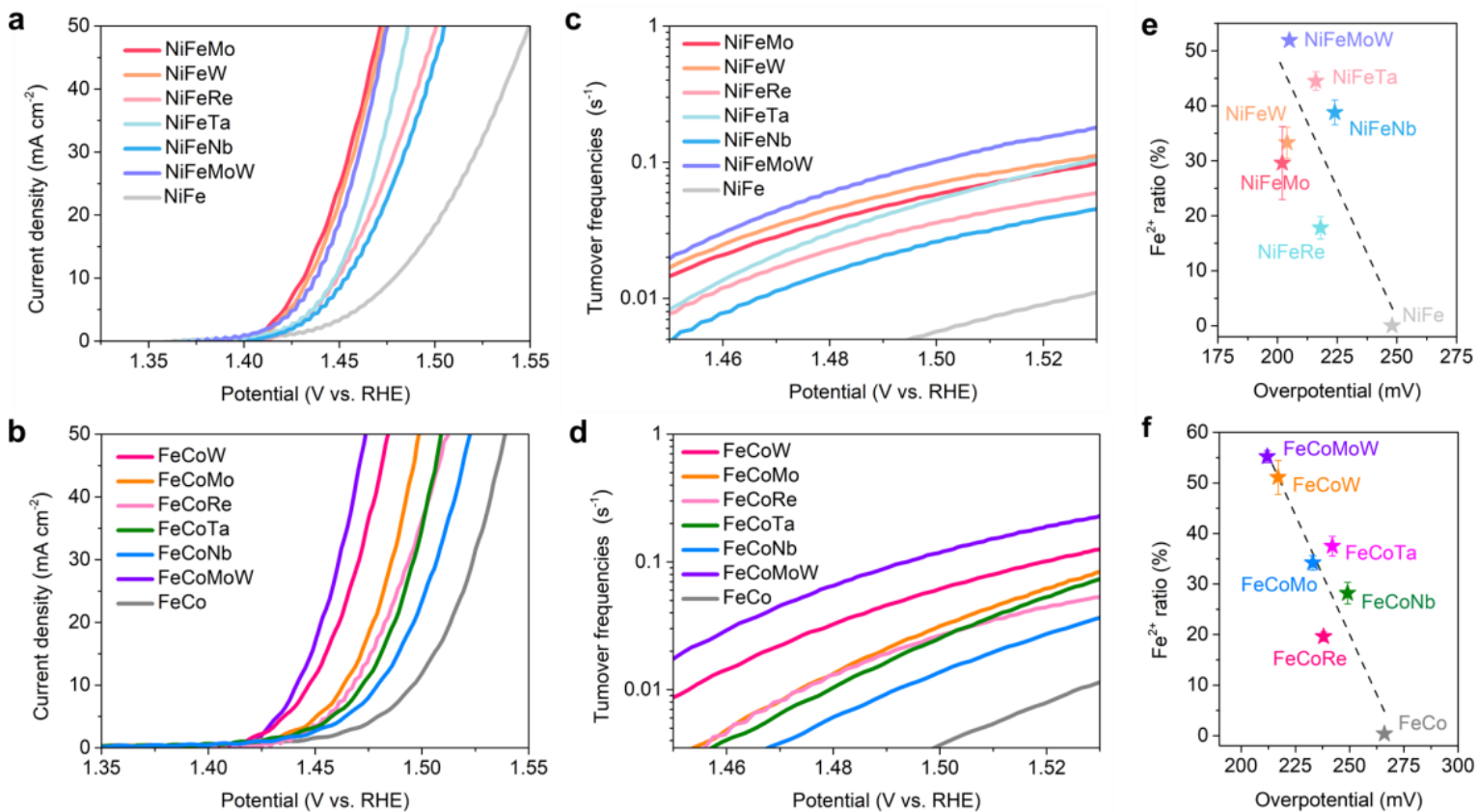

Figure 3. Performance of NiFeX and FeCoX catalysts in $1 \mathrm{M}$ KOH electrolyte at $25^{\circ} \mathrm{C}$. (a, b) OER polarization curves on carbon paper measured with a $5 \mathrm{mV} \cdot \mathrm{s}^{-1}$ scan rate, with $95 \% i R$ correction. (c, d) Turnover frequency trends as a function of potential for catalysts on carbon paper measured with a $5 \mathrm{mV} \cdot \mathrm{s}^{-1}$ scan rate, with $95 \% i R$-correction. (e, f) Overpotentials at current density $10 \mathrm{~mA} \cdot \mathrm{cm}^{-2}$ tested vs. $\mathrm{Fe}^{2+}$ ratio. 

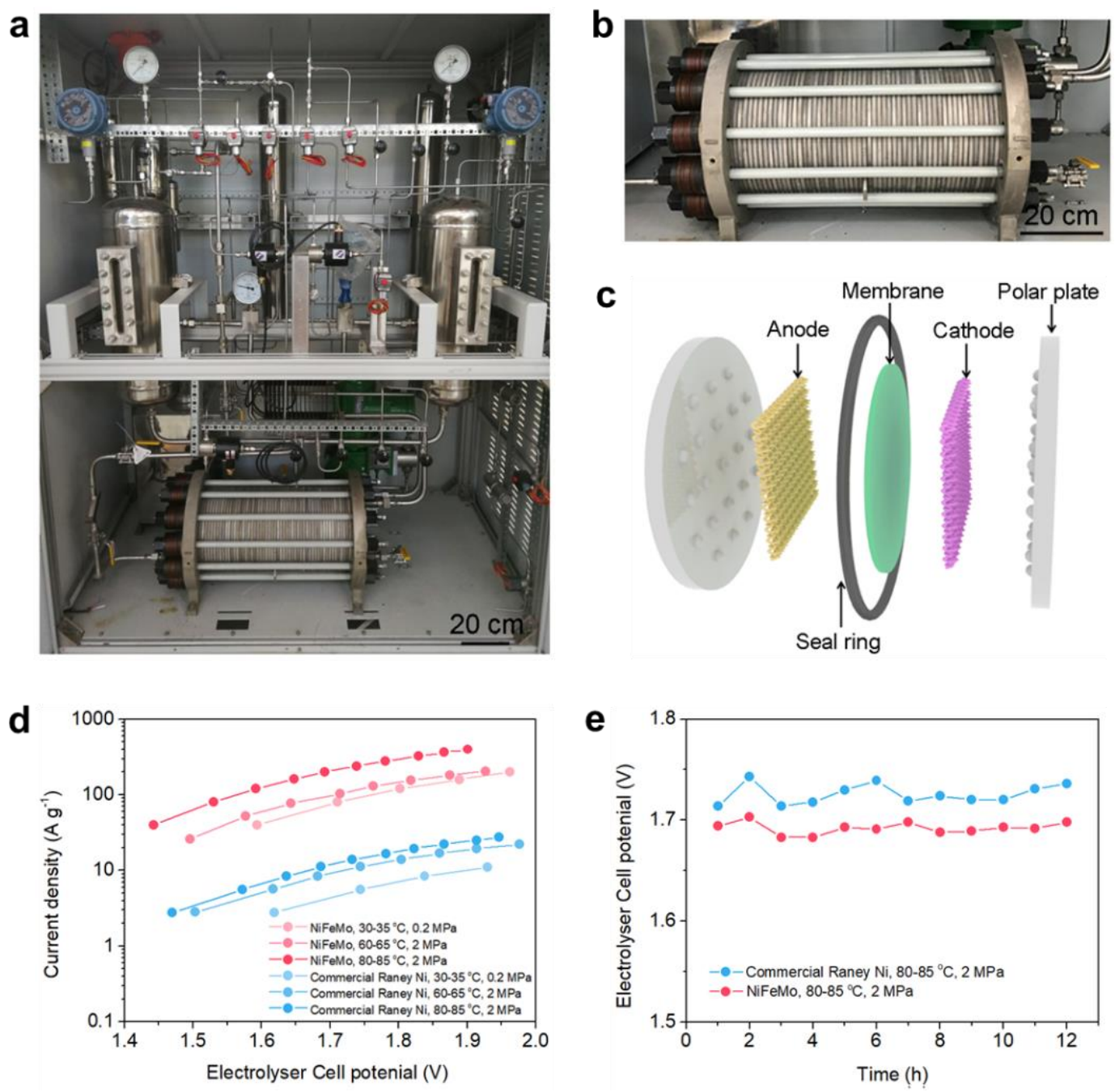

Figure 4. Performance of NiFeMo catalysts in industrial electrolyzer systems. (a) Photograph of an industrial electrolyzer device. (b) Zoom-in of the industrial electrolyzer. (c) Schematic illustration of the structure of an electrolyzer cell. (d) Polarization curves measured during water electrolysis using NiFeMo electrode (red) and commercial Raney Ni electrode (blue) as an anode, respectively, and commercial Ru electrode as a cathode. (e) The cell voltage of the electrolyzer held at $300 \mathrm{~mA} \cdot \mathrm{cm}^{-2}$ for $12 \mathrm{~h}$ at $80-85^{\circ} \mathrm{C}, 2 \mathrm{MPa}$. 